\title{
A Note on the VLF Emissions in the Outer Exosphere
}

Ellis (1957, 1959), Gendrin (1961), Gintsburg (1961), McInnes (1961), Ondoh (1961), and Sturrock (1962) have proposed the Čerenkov emissions from charged particles moving along geomagnetic line of force in the exosphere in order to explain the VLF emissions.

In this paper we examine a possibility of amplification of Čerenkov instability in the outer exosphere suggested by Ondoh (1962 a, b).

At geocentric distances beyond about 4 earth radii $\left(2 \frac{f_{0}}{f_{H}} \frac{v}{c}\right)^{2} \geqq 1$ for velocity $v$ of the order of $10^{4} \mathrm{~km} / \mathrm{s}$, where $f_{0}$ is the electron plasma frequency, $f_{H}$ the electron gyrofrequency, $v$ the velocity of charged particles, and $c$ the velocity of light (Gallet, 1959).

Hence the Cerenkov instability with the complex frequency $f=f_{r} \pm i r$ may appear in the outer exosphere, where $f_{r}=\frac{1}{2} f_{H} \cos \theta, r=\frac{1}{2} f_{H} \cos \theta\left\{\left(2 \frac{f_{0}}{f_{H}} \frac{v}{c} \frac{1}{\sqrt{1-\frac{v^{2}}{c^{2}} \cos ^{2} \theta}}\right)^{2}-1\right\}^{1 / 2}$, and $\theta$ the angle between the wave normal direction and the velocity direction of charged particles moving along geomagnetic line of force.

The expression $\left(2 \frac{f_{0}}{f_{H}} \frac{v}{c}\right)^{2}>1$ is rewritten as $v>\frac{c f_{H}}{2 f_{0}} \cdots \cdots . . .(1)$.

At the frequency of $\frac{1}{2} f_{H} \cos \theta$ the refractive index of extraordinary waves $n$ becomes $\left[1+\frac{f_{0}^{2}}{f\left(f_{H} \cos \theta-f\right)}\right]^{1 / 2}=\left(1+\frac{4 f_{0}^{2}}{f_{H}{ }^{2} \cos ^{2} \theta}\right)^{1 / 2}$. Then we have $n \simeq \frac{2 f_{0}}{f_{H} \cos \theta} \cdots \cdots$ for the frequency of $\frac{1}{2} f_{H} \cos \theta$ in the outer exosphere since $\frac{4 f_{0}^{2}}{f_{H}^{2} \cos ^{2} \theta} \gg 1$ in the region.

Using the expressions (1) and (2) we have an inequality of $\cos \theta>\frac{c}{n v}$ that is the condition for the anomalous Doppler effect shown by Ginzburg (1959).

Thus the instability suggested by Ondoh (1962 a, b) may be the anomalous Doppler effect.

The anomalous Doppler waves are growing waves (Ginzburg, 1959).

Therefore the Čerenkov instability may produce the growing waves.

Considering penetrations of charged particles with velocity of $v_{1}=\frac{C f_{H}}{2 f_{0}}$ and $v_{2}>\frac{C f_{H}}{2 f_{0}}$ into the outer exosphere simultaneously, we may explain the VLF emissions.

\section{References}

Ellis G.R.A. (1957) J. Atmos. Terr. Phys. 10, 302.

Ellis G.R.A. (1959) Planet. Space Sci. 1, 256.

Gallet R.M. (1959) Proc. IRE 47, 221.

Gendrin R. (1961) Planet. Space Sci. 5, 274.

Ginzburg V.L. (1959) Usp. Fiz. Nauk 69, 537.

Gintsburg M.A. (1961) Phys. Rev. Letters 7, 399.

McInnes B.A. (1961) Austr. J. Phys. 14, 218. 
Ondoh T. (1961) J. Geomag. Geoelect. Kyoto 11, 77.

Ondoh T. (1962a) Planet. Space Sci. 9, 69.

Ondoh T. (1962b) J. Geomag. Geoelect. Tokyo Vol. 14, No. 2.

Sturrock P.A. (1962) J. National Bureau of Standards 66D, 153.

\section{By Tadanori ONDOH}

Geophysical Institute, Kyoto University, Kyoto. 